\title{
Paraneoplastic syndromes and inflammatory rheumatic diseases : Not everything that glitters is gold. The case of polymyalgia rheumatica.
}

\author{
Ciro Manzo* \\ Mariano Lauro Hospital, Sant'Agnello, Italy
}

Accepted on 06 August 2018

\section{Editorial}

The term "Paraneoplastic Syndromes" (P.S.) refers to all the signs and symptoms caused by cancer but which are not directly related to the tumor mass nor its metastases. It is estimated that up to $8 \%$ of patients with cancer may present a P.S. Its manifestations may appear at the same time, before or after cancer, and, by definition, they regress after its treatment [1].

Paraneoplastic rheumatologic syndromes have been repeatedly descripted in the literature, especially for dermatomyositis and vasculitis $[2,3]$. They are thought to be caused by a disturbance of the immune response [4,5] or to be a consequence of abnormal cytokine release (typical the case of the Vascular Endothelial Growth Factor, VEGF). When paraneoplastic rheumatologic syndromes are not synchronous to cancer, most investigators agree in highlighting that the expression of an occult cancer must become clinically evident within a determinate timeframe, that is no more than 2 years. Paraneoplastic rheumatologic syndromes are difficultly distinguishable from idiopathic, non paraneoplastic manifestations, even if are usually less responsive to therapy than are the idiopathic ones [4]. On the other hand, the patients with inflammatory rheumatic diseases have an increased risk of developing malignancies [6]. In fact, chronic inflammation per se is an important favoring factor : for example, in patients with Rheumatoid Arthritis (RA), Sjogren's Syndrome (SS) or Systemic Lupus Erythematosus (SLE), cancer risk is strictly related to disease activity [7]. Instead, the pathogenetic importance of the immunosuppressive and/or immunoregulatory drugs is not univocal $[8,9]$.

The question as to whether an inflammatory rheumatic disease is a paraneoplastic syndrome or a differential diagnosis of malignancy problematic is anything but trivial. This is particularly intriguing for less specific findings, such as joint pain, or constitutional manifestations (i.e., fever, loss of appetite, weight loss, fatigue, general malaise). The possibility that cancer can favor the production of autoantibodies, identical to those present in some rheumatic diseases, is another confounding element [10].

The question is of particular relevance for Polymyalgia Rheumatica (PMR). PMR can be considered the most frequent inflammatory rheumatic disease in Caucasian persons older than 70 years [11]. Its diagnosis is based upon recognition of a clinical syndrome consisting of pain and stiffness in the shoulder and pelvic girdle and morning stiffness lasting at least 45 minutes [12]. Constitutional manifestations may be present. In absence of a specific diagnostic test, the diagnosis of PMR is basically clinical $[12,13]$. The rapid response to low-dosed glucocorticoids (GCs) ( $<20 \mathrm{mg}$ prednisone per day), represents an additional diagnostic confirmation element. However, several patients fail to achieve a complete response after few days and - on the other hand - some diseases can mimic PMR not only in the clinical features but also in a fast response to low-dosed systemic GCs. Some of these diseases fail to maintain the first positive response in a short time but others (solid or hematological tumors, for example) can do it [14]. Furthermore, some patients initially diagnosed with PMR may be reclassified as having a different disease during follow-ups, seronegative RA being the most common alternative $[13,14]$.

In the clinical practice, we can have three diagnostic challenges:

1) The diagnosis of PMR is not correct. Failure to respond to GCs therapy or frequent relapses must be considered as elements of suspicion. Furthermore, the presence of not typical laboratory findings should also be considered as a warning: among these, macrocytic anemia [15] or bicytopenia [16]. In these cases, the diagnosis should be revised. Furthermore, PMR symptoms may also occur in patients with cancer [17]. In a systematic review recently published by Muller et al. [18], amongst other important topics, a short-term association between PMR and cancer (less than 6 to 12 months after PMR diagnosis) deserved important considerations. In these cases, PMR may represent a misdiagnosis (the patient had cancer and was diagnosed with a PMR), or it is possible that the two conditions coexist. According to the guideline suggestions regarding exclusion cancer before making the PMR diagnosis, PMR can be considered as a PS only when cancer is synchronous or following the diagnosis ;

2) During follow-up, PMR is reclassified as a different disease. Among older patients initially identified as PMR and evaluated extensively since the onset, more than $20 \%$ are subsequently reclassified as seronegative RA. Seronegative RA is the most common diagnostic alternative, but not the only one [14]. In these cases, the significance for PMR as a P.S. must be identified with that of the subsequently recognized disease. Seronegative remitting symmetrical synovitis with pitting edema (RS3PE) deserves separate considerations. RS3PE is an uncommon elderly-onset rheumatic condition, described for the first time by McCarty et al. in 1985, characterized by tenosynovitis of extensor tendons at the wrist and (less frequently) at the feet, rapidly responding to low-dosed GCs (5-10 mg prednisone for day, on average). The levels of VEGF are significantly higher in these patients than in controls, and its levels decreased after GCs treatment. It is estimated that no more than $10 \%$ of patients with PMR may have RS3PE. The 
Citation: Manzo C. Paraneoplastic syndromes and inflammatory rheumatic diseases : Not everything that glitters is gold. The case of polymyalgia rheumatica. J Med Oncl Ther 2018;3(2):21-22.

association RS3PE + PMR must be considered a neoplastic warning $[19,20]$;

3) The diagnosis of PMR is correct and no alternative diagnosis is found during follow-up. A rigorous diagnostic work-up must be performed before making the PMR diagnosis. This diagnostic work-up must consider some inexpensive tests such as a chest X-ray, a prostate specific antigen, an abdominal-pelvic and breast (in women) ultrasound examination. In patients with highest risk of having an underlying cancer, additional tailored investigations should be requested [21]. When cancer is then diagnosed during the follow-up of these patients, PMR can be considered a real P.S. .

Not all that glitters is gold, one might say.

\section{Conflict of interest}

The author declare no conflict of interest.

\section{References}

1. Pelosof LC, Gerber DE. Paraneoplastic syndromes : An approach to diagnosis and treatment. Mayo Clin Proc. 2010;85:838-54.

2. Leatham H, Schadt C, Chisolm S, et al. Evidence supports blind screening for internal malignancy in dermatomyositis: Data from 2 large US dermatology cohorts. Medicine (Baltimore). 2018;97:e9639.

3. Azar L, Khasnis A. Paraneoplastic rheumatologic syndromes. Curr Opin Rheumatol. 2013;25:44-9.

4. Racanelli V, Prete M, Minoia C, et al. Rheumatic disorders as paraneoplastic syndromes. Autoimmun Rev. 2008;7:352-8.

5. Manzo C. Autoimmunity and cancer : A lesson from systemic sclerosis. J Med Oncl Ther. 2018;3:1-2.

6. Giat E, Ehrenfeld M, Shoenfeld Y. Cancer and autoimmune diseases. Autoimmun Rev. 2017;16:1049-57.

7. Cutolo M, Paolino S, Pizzorni C. Possible contribution of chronic inflammation in the induction of cancer in rheumatic diseases. Clin Exp Rheumatol. 2014;32:839-47.

8. Georgescu L, Quinn GC, Schwartzman S, et al. Lymphoma in patients with rheumatoid arthritis :Association with the disease state or methotrexate treatment. Semin Arthritis Rheum. 1997;26:794-804.

9. Chen Y, Friedman M, Liu G, et al. Do tumor necrosis factor inhibitors increase cancer risk in patients with chronic immune-mediated inflammatory disorders? Cytokine. 2018;101:78-88.

10. Madrid FF, Maroun MC. Serologic laboratory findings in malignancy. Rheum Dis Clin North Am. 2011;37:4.
11. Sobrero A, Manzo C, Stimamiglio A. The role of the general practitioner and the out-of-hospital public rheumatologist in the diagnosis and follow-up of patients with polymyalgia rheumatica. Reumatismo. 2018;70:44-50.

12. Dasgupta B, Cimmino MA, Maradit-Kremers H, et al. Provisional classification criteria for polymyalgia rheumatica: A European league against rheumatism/ American college of rheumatology collaborative initiative. Ann Rheum Dis. 2012;71:484-92.

13. Manzo C, Natale M, Traini E. Diagnosis of polymyalgia rheumatica in primary health care : Favoring and confounding factors- a cohort study. Reumatologia. 2018; 56:131-39.

14. Manzo C., Camellino D. Polymyalgia Rheumatica (PMR): Diagnostic and therapeutic issues of an apparently straightforward disease. Recenti Prog Med. 2017;108:221-31.

15. Manzo C. About a gastric neuroendocrine tumor presenting as polymyalgia rheumatica and macrocytic anemia. J Clin Case Rep. 2016;7:784.

16. Anton E. More on polymyalgia rheumatica (PMR) as a paraneoplastic rheumatic syndrome in the elderly (bicytopenia and PMR preceding acute myeloid leukemia). J Clin Rheumatol.2007;13:114.

17. Hashefi M. Rheumatologic manifestations of malignancy. Clin Geriatr Med. 2017; 33:73-86.

18. Muller S, Hider S, Helliwell T, et al. The real significance for polymyalgia rheumatica as a paraneoplastic syndrome. Reumatismo. 2018; 70:23-34.

19. Manzo C. The paraneoplastic meaning of R3SPE (remitting seronegative simmetrical synovitis with pitting edema) syndrome. J Med Oncl Ther. 2016;1:72-5.

20. Manzo C, Natale M. Polymyalgia Rheumatica in Association with Remitting Seronegative Sinovitis with Pitting Edema: a Neoplastic Warning. Can Geriatr J. 2017;20:94-6.

21. Manzo C, Natale M. Polymyalgia rheumatica and cancer risk: The importance of the diagnostic set. Open Access Rheumatol. 2016;8:93-5.

\section{*Correspondence to:}

Ciro Manzo, MD,

Mariano Lauro hospital,

Sant'Agnello, Italy,

E-mail: cirmanzo@libero.it 